\title{
Colpo di scena
}

Giovanni Lodi

Dipartimento di Scienze Biomediche, Chirurgiche e Odontoiatriche

Università degli Studi di Milano

via Beldiletto 1/3 Milano 20142

Italia

giovanni.lodi@unimi.it

tel. + 390250319021

fax. +390250319041

Muovendomi senza uno scopo preciso tra gli oltre 28 milioni di referenze che formano PubMed (ognuno ha i propri guilty pleasures), mi sono imbattuto in un articolo di cui vi voglio raccontare. Si tratta di una revisione sistematica in cui gli autori hanno provato a capire quali siano le aspettative dei pazienti nei confronti di test diagnostici e trattamenti medici o chirurgici. I risultati mostrano in maniera inequivocabile un eccesso di ottimismo. Una parte cospicua dei pazienti (fino a 9 su 10) si attende dagli interventi e dagli esami a cui si sottopone benefici maggiori di quanto sia legittimo aspettarsi, sottovalutando con simile frequenza i rischi a essi associati.

Questo probabilmente vale anche per l'odontoiatria, se più di uno studio ha svelato le irrealistiche aspettative che i pazienti nutrono nei confronti, ad esempio, della terapia implantare.

Se pensiamo al paziente che oggi ci guardava come se fossimo la piscina di Lourdes o a quello che ieri ha minacciato di portarci in tribunale perché il risultato dei nostri trattamenti non è esattamente ciò che si aspettava, la cosa non ci stupisce. Però quei dati scritti nero su bianco, in un'autorevole rivista di medicina generale, fanno un altro effetto. Da dove nasce tanto ottimismo?

Forse dalla rappresentazione offerta dai mass media delle magnifiche sorti e progressive della medicina? 0 non sarà piuttosto un problema di comunicazione? È possibile che medici e odontoiatri non siano abbastanza bravi nello spiegare ai pazienti i reali benefici e i potenziali danni di quanto prescrivono? Con buona pace di consenso informato e alleanza terapeutica.

E invece ecco il colpo di scena. A distanza di pochi mesi gli stessi autori pubblicano una nuova revisione sistematica molto simile alla prima, ma che studia i medici. Il risultato? Stesso ottimismo, stesse irrealistiche aspettative, questa volta però molto meno giustificate. Che forse aiutano a capire i pazienti, ma che rendono la situazione nel suo complesso ancora più complicata.

Buona lettura 